Geometry 85 Topology

Volume 7 (2003) 1055-1073

Published: 29 December 2003

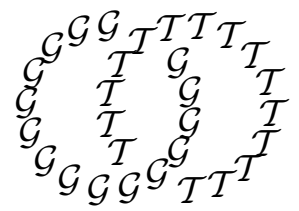

\title{
An infinite family of tight, not semi-fillable contact three-manifolds
}

\author{
PAOLO Lisca \\ ANDRÁs I STIPSICZ \\ Dipartimento di Matematica, Università di Pisa \\ I-56127 Pisa, ITALY \\ and \\ Rényi Institute of Mathematics, Hungarian Academy of Sciences \\ H-1053 Budapest, Reáltanoda utca 13-15, Hungary \\ Email: lisca@dm.unipi.it and stipsicz@math-inst.hu
}

\begin{abstract}
We prove that an infinite family of virtually overtwisted tight contact structures discovered by Honda on certain circle bundles over surfaces admit no symplectic semi-fillings. The argument uses results of Mrowka, Ozsváth and Yu on the translation-invariant solutions to the Seiberg-Witten equations on cylinders and the non-triviality of the Kronheimer-Mrowka monopole invariants of symplectic fillings.
\end{abstract}

AMS Classification numbers Primary: 57R57

Secondary: 57R17

Keywords: Tight, fillable, contact structures

Proposed: Tomasz Mrowka

Seconded: Yasha Eliashberg, John Morgan
Received: 4 September 2002

Accepted: 23 December 2003

(c) Geometry $\mathcal{G}$ Topology $\mathcal{P}$ ublications 


\section{Introduction}

Let $Y$ be a closed, oriented three-manifold. A positive, coorientable contact structure on $Y$ is the kernel $\xi=\operatorname{ker} \alpha \subset T Y$ of a one-form $\alpha \in \Omega^{1}(Y)$ such that $\alpha \wedge d \alpha$ is a positive volume form on $Y$. In this paper we only consider positive, coorientable contact structures, so we call them simply 'contact structures'. For an introduction to contact structures the reader is referred to [1, Chapter 8] and $[8]$.

There are two kinds of contact structures $\xi$ on $Y$. If there exists an embedded disk $D \subset Y$ tangent to $\xi$ along its boundary, $\xi$ is called overtwisted, otherwise it is said to be tight. The isotopy classification of overtwisted contact structures coincides with their homotopy classification as tangent two-plane fields [5]. Tight contact structures are much more difficult to classify, and capture subtle information about the underlying three-manifold.

A contact three-manifold $(Y, \xi)$ is symplectically fillable, or simply fillable, if there exists a compact symplectic four-manifold $(W, \omega)$ such that (i) $\partial W=Y$ as oriented manifolds (here $W$ is oriented by $\omega \wedge \omega$ ) and (ii) $\left.\omega\right|_{\xi} \neq 0$ at every point of $Y$. $(Y, \xi)$ is symplectically semi-fillable if there exists a fillable contact manifold $(N, \eta)$ such that $Y \subset N$ and $\left.\eta\right|_{Y}=\xi$. Semi-fillable contact structures are tight [6]. The converse is known to be false by work of Etnyre and Honda, who recently found two examples of tight but not semi-fillable contact three-manifolds [9]. This discovery naturally led to a search for such examples, in the hope that they would tell us something about the difference between tight and fillable contact structures. By a result announced by E Giroux [12], isotopy classes of contact structures on a closed three-manifold are in one-toone correspondence with "stable" isotopy classes of open book decompositions. When the monodromy of the open book decomposition is positive, the corresponding contact structure is fillable. Therefore, it would be very interesting to know examples of monodromies associated with tight but not fillable contact structures.

In this paper we prove that infinitely many tight contact circle bundles over surfaces are not semi-fillable. Let $\Sigma_{g}$ be a closed, oriented surface of genus $g \geq 1$. Denote by $Y_{g, n}$ the total space of an oriented $S^{1}$-bundle over $\Sigma_{g}$ with Euler number $n$. Honda gave a complete classification of the tight contact structures on $Y_{g, n}$ [15]. The three-manifolds $Y_{g, n}$ carry infinitely many tight contact structures up to diffeomorphism. The classification required a special effort for two tight contact structures $\xi_{0}$ and $\xi_{1}$, for $n, g$ satisfying $n \geq 2 g$ (see Definition 2.5 below). Honda conjectured that none of these contact structures 
are symplectically semi-fillable. Our main result establishes Honda's conjecture in infinitely many cases:

Theorem 1.1 Suppose that $d(d+1) \leq 2 g \leq n \leq(d+1)^{2}+3$ for some positive integer $d$. Then, the tight contact structures $\xi_{0}$ and $\xi_{1}$ on $Y_{g, n}$ are not symplectically semi-fillable.

Remark 1.2 Let $\xi$ be a tight contact structure on a three-manifold $Y$. If the pull-back of $\xi$ to the universal cover of $Y$ is tight, then $\xi$ is called universally tight. Honda showed in [15] that the contact structures $\xi_{i}$ become overtwisted when pulled-back to a finite cover, i.e. they are virtually overtwisted. Thus, the question whether every universally tight contact structure is symplectically fillable is untouched by Theorem 1.1.

The proof of Theorem 1.1 is similar in spirit to the argument used by the first author to prove that certain oriented three-manifolds with positive scalar curvature metrics do not carry semi-fillable contact structures [17, 18]. But the fact that the three-manifolds $Y_{g, n}$ do not admit positive scalar curvature metrics made necessary a modification of the analytical as well as the topological parts of the original argument.

We first show that if $W$ is a semi-filling of $\left(Y_{g, n}, \xi_{i}\right)$, then $\partial W$ is connected, $b_{2}^{+}(W)=0$ and the homomorphism $H^{2}(W ; \mathbb{R}) \rightarrow H^{2}(\partial W ; \mathbb{R})$ induced by the inclusion $\partial W \subset W$ is the zero map (see Proposition 4.2). To do this, we start by identifying the $\operatorname{Spin}^{c}$ structures $\mathbf{t}_{\xi_{i}}$ induced by the contact structures $\xi_{i}$. Then, using results of Mrowka, Ozsváth and $\mathrm{Yu}$ [20], we establish properties of the Seiberg-Witten moduli spaces for the Spin ${ }^{c}$ structures $\mathbf{t}_{\xi_{i}}$ which are sufficient to apply the argument used in the positive scalar curvature case. Such an argument relies on the non-triviality of the Kronheimer-Mrowka monopole invariants of a symplectic filling [16].

Then, under some restrictions on $g$ and $n$, we construct smooth, oriented fourmanifolds $Z$ with boundary orientation-reversing diffeomorphic to $Y_{g, n}$, with the property that if $W$ were a symplectic filling of $\left(Y_{g, n}, \xi_{i}\right)$, the closed fourmanifold $W \cup_{Y_{g, n}} Z$ would be negative definite and have non-diagonalizable intersection form. On the other hand, by Donaldson's celebrated theorem [2, 3], such a closed four-manifold cannot exist. Therefore, $\left(Y_{g, n}, \xi_{i}\right)$ does not have symplectic fillings.

The paper is organized as follows. In Section 2 we define, following [15], the contact structures $\xi_{0}$ and $\xi_{1}$. In Section 3 we determine the $\operatorname{Spin}^{c}$ structures $\mathbf{t}_{\xi_{i}}$, and in Section 4 we prove Theorem 1.1. 
Acknowledgements The first author is grateful to Peter Ozsváth for enlighting e-mail correspondence. The first author was partially supported by MURST, and he is a member of EDGE, Research Training Network HPRN-CT2000-00101, supported by The European Human Potential Programme. The second author was partially supported by Bolyai Ösztöndíj and OTKA T34885.

\section{Definition of the contact structures}

In this section we describe in detail the construction of the contact structures $\xi_{i}$. For the sake of the exposition, we start by recalling some basic facts regarding convex surfaces and Legendrian knots in contact three-manifolds.

\section{Basic properties of contact structures}

Let $Y$ be a closed, oriented three-manifold and let $\xi$ be a contact structure on $Y$.

Definition 2.1 An embedded surface $\Sigma \subset Y$ is convex if there exists a vector field $V$ on $Y$ such that (i) $V$ is transverse to $\Sigma$ and (ii) $V$ is a contact vector field, i.e. $\xi$ is invariant under the flow generated by $V$. The dividing set is

$$
\Gamma_{\Sigma}(V)=\{p \in \Sigma \mid V(p) \in \xi\} \subset \Sigma .
$$

The following facts are proved in the seminal paper by E Giroux [10]:

(1) Let $\Sigma \subset(Y, \xi)$ be an embedded surface. Then, $\Sigma$ can be $C^{\infty}$-perturbed to a convex surface.

(2) Let $\Sigma \subset(Y, \xi)$ be a convex surface and $V$ a contact vector field transverse to $\Sigma$. Then, (i) the isotopy class $\Gamma_{\Sigma}$ of $\Gamma_{\Sigma}(V)$ does not depend on the choice of $V$ and (ii) the germ of $\xi$ around $\Sigma$ is determined by $\Gamma_{\Sigma}$.

In the case of a convex torus $T \subset(Y, \xi)$, the set $\Gamma_{T}$ consists of an even number of disjoint simple closed curves. The germ of $\xi$ around $T$ is determined by the number of connected components of $\Gamma_{T}$ - the dividing curves - together with a (possibly infinite) rational number representing their slope with respect to an identification $T \cong \mathbb{R}^{2} / \mathbb{Z}^{2}$. Note that the slope depends on the choice of the identification. If $T$ is the boundary of a neighborhood of a knot $k \subset Y$, then by identifying the meridian with one copy of $\mathbb{R} / \mathbb{Z}$ in the above identification, 
the slope $p / q$, regarded as a vector $\left(\begin{array}{l}q \\ p\end{array}\right)$, is determined up to the action of the group

$$
\left\{\left(\begin{array}{cc}
1 & m \\
0 & 1
\end{array}\right) \mid m \in \mathbb{Z}\right\}
$$

A knot $k \subset(Y, \xi)$ is Legendrian if $k$ is everywhere tangent to $\xi$. The framing of $k$ naturally induced by $\xi$ is called the contact framing. A Legendrian knot $k$ has a basis $\left\{U_{\alpha}\right\}$ of neighborhoods with convex boundaries. The dividing set of each boundary $\partial U_{\alpha}$ consists of two dividing curves having the same slope independent of $\alpha$. Any one of those neighborhoods of $k$ is called a standard convex neighborhood of $k$. The meridian and the contact framing of a Legendrian knot $k \subset(Y, \xi)$ provides an identification of the convex boundary $T$ of a neighborhood of $k$ with $\mathbb{R}^{2} / \mathbb{Z}^{2}$; easy computation shows that with this identification the slope of the dividing curves is $\infty$.

Let $\Sigma_{g}$ be a closed, oriented surface of genus $g \geq 1$, and let $\pi: Y_{g, n} \rightarrow \Sigma_{g}$ be an oriented circle bundle over $\Sigma_{g}$ with Euler number $n$. Let $\xi$ be a contact structure on $Y_{g, n}$ such that a fiber $f=\pi^{-1}(s) \subset Y_{g, n}\left(s \in \Sigma_{g}\right)$ is Legendrian. We say that $f$ has twisting number -1 if the contact framing of $f$ is ' -1 ' with respect to the framing determined by the fibration $\pi$. A contact structure on $Y_{g, n}$ is called horizontal if it is isotopic to a contact structure transverse to the fibers of $\pi$.

\section{Definition of the contact structures $\xi_{i}$}

The following lemma is probably well-known to the experts. A proof can be found e.g. in $[11, \S 1 . D]$. We include it here to make our exposition more selfcontained, and for later reference.

Lemma 2.2 The circle bundle $\pi: Y_{g, 2 g-2} \rightarrow \Sigma_{g}$ carries a horizontal contact structure $\zeta$ such that all the fibers of $\pi$ are Legendrian and have twisting number -1 .

Proof Think of $Y_{g, 2 g-2}$ as the manifold of the oriented lines tangent to $\Sigma_{g}$. Then, the fiber $\pi^{-1}(s) \subset Y_{g, 2 g-2}\left(s \in \Sigma_{g}\right)$ consists of all the oriented lines $l$ tangent to $\Sigma_{g}$ at the point $s$. The contact two-plane $\zeta(l)$ at the point $l$ is, by definition, the preimage of $l \subset T_{s} \Sigma_{g}$ under the differential of $\pi$. It is a classical fact that $\zeta$ is a contact structure. It follows directly from the definition that every fiber of $\pi$ is Legendrian and has twisting number -1 . To see that $\zeta$ is horizontal, let $V$ be a vector field on $Y_{g, 2 g-2}$ tangent to $\zeta$, 
transverse to the fibers and such that, for every $l \in Y_{g, 2 g-2}$ the projection $d \pi(V(l)) \in l \subset T_{\pi(l)} Y_{g, 2 g-2}$ defines the orientation on $l$. Let $\lambda$ be a one-form defining $\zeta$, and $T$ a nonvanishing vector field on $Y_{g, 2 g-2}$ tangent to the fibers. The fact that $d \lambda$ does not vanish on the contact planes is equivalent to the fact that the Lie derivative of $\lambda$ in the direction of $V$ is nowhere vanishing when evaluated on $T$, because $\mathfrak{L}_{V}(\lambda)(T)=d \lambda(V, T)$. Thus, following the flow of $V$ the contact structure $\zeta$ can be isotoped to a transverse contact structure.

Suppose that $n \geq 2 g$. Let $\zeta$ be the contact structure given by Lemma 2.2 (according to $[15, \S 5] \zeta$ could be any horizontal contact structure on $Y_{g, 2 g-2}$ such that a fiber $f$ of the projection $\pi$ is Legendrian with twisting number $-1)$. Let $U \subset Y_{g, 2 g-2}$ be a standard convex neighborhood of $f$. The fibration induces a trivialization $U \cong S^{1} \times D^{2}$. Remove $U$ from $Y_{g, 2 g-2}$ and reglue it using the diffeomorphism $\varphi_{A}: \partial U \rightarrow-\partial\left(Y_{g, 2 g-2} \backslash U\right)$ determined, via the above trivialization, by the matrix

$$
A=\left(\begin{array}{cc}
-1 & 0 \\
p+1 & -1
\end{array}\right)
$$

where $p=n-2 g+1$. The map $\pi$ extends to the resulting three-manifold yielding the bundle $Y_{g, n}$, and we are going to show that $\zeta$ extends as well. The germ of $\zeta$ around $\partial\left(Y_{g, 2 g-2} \backslash U\right)$ is determined by the slope of any dividing curve $C \subset \partial\left(Y_{g, 2 g-2} \backslash U\right)$. We are going to extend $\zeta$ to $U$ as a tight contact structure $\eta$ having convex boundary and two dividing curves isotopic to $\varphi_{A}^{-1}(C) \subset \partial U$. Since the fiber $f$ has twisting number -1 with respect to $\zeta$, the slope of $C$ is -1 with respect to the trivialization used to define the gluing map. Therefore, the slope of $\varphi_{A}^{-1}(C)$ is $p$. Applying the self-diffeomorphism of $S^{1} \times D^{2}$ given by the matrix $\left(\begin{array}{rr}1 & -1 \\ 0 & 1\end{array}\right)$, we may assume that the boundary slope of $(U, \eta)$ be $-\frac{p}{p-1}$.

Summarizing, assuming the existence of $\eta$ we have constructed a contact threemanifold $\left(Y_{g, n}, \xi\right)$ of the form

$$
\left(Y_{g, n}, \xi\right)=\left(Y_{g, 2 g-2} \backslash U, \zeta\right) \cup_{\varphi_{A}}(U, \eta) .
$$

The following result says that we have two possible choices for $\eta$ when $n>2 g$, and one when $n=2 g$.

Theorem 2.3 [14] Let $p$ be a positive integer. Up to an isotopy keeping the boundary fixed, there are at most two tight contact structures on $S^{1} \times D^{2}$ with convex boundary and two dividing curves with slope $-\frac{p}{p-1}$. More precisely, when $p>1$ there are exactly two such contact structures. When $p=1$, i.e. when the boundary slope is infinite, there is only one. 
We need to be more specific about the contact structures appearing in the statement of Theorem 2.3. The next lemma will be used in the following section as well.

Lemma 2.4 Let $p$ be a positive integer and $\eta$ a tight contact structure on $S^{1} \times D^{2}$ with convex boundary and two dividing curves with slope $-\frac{p}{p-1}$. Then, $\eta$ can be isotoped keeping the boundary convex until there exists a section $v$ of $\eta$ such that:

(1) At every boundary point $v$ is nonvanishing and tangent to the circles $S^{1} \times\{x\}, x \in \partial D^{2}$

(2) The zero locus of $v$ is a smooth curve homologous to

$$
\pm(p-1)\left[S^{1} \times\{(0,0)\}\right] .
$$

Moreover, each sign in formula (2.2) above can be realized by some contact structure $\eta$.

Proof Using Giroux's Flexibility Theorem (see [10] and $[14, \S 3.1 .4]$ ) we may isotope $\eta$ keeping the boundary convex until $\eta$ is tangent to the circles $S^{1} \times\{x\}$, $x \in \partial D^{2}$ at each boundary point.

By [14, Proposition 4.15] there exists a decomposition

$$
S^{1} \times D^{2}=N \cup\left(S^{1} \times D^{2} \backslash N\right),
$$

where $N$ is a standard convex neighborhood of a Legendrian knot isotopic to the core circle of $S^{1} \times D^{2}$. Thus, $N \cong S^{1} \times D^{2}$ with coordinates $(z,(x, y))$ and

$$
\left.\eta\right|_{N}=\operatorname{ker}(\sin (2 \pi z) d x-\cos (2 \pi z) d y) .
$$

Moreover, there is a diffeomorphism

$$
\varphi: S^{1} \times D^{2} \backslash N \cong T^{2} \times[0,1]
$$

and $\left(T^{2} \times[0,1], \varphi_{*} \eta\right)$ is a basic slice (see $\left.[14, \S 4.3]\right)$ with convex boundary components $T^{2} \times\{0\}$ and $T^{2} \times\{1\}$ of slopes -1 and $-\frac{p}{p-1}$ respectively. Without loss of generality we may also assume that

$$
\left.\varphi\right|_{S^{1} \times \partial D^{2}}: S^{1} \times \partial D^{2} \rightarrow T^{2} \times\{1\}
$$

is the obvious identification.

According to [14, Lemma 4.6 and Proposition 4.7] the Euler class of a basic slice with boundary slopes 0 and -1 , relative to a section which is nowhere zero at the boundary and tangent to it, is equal to

$$
\pm(0,1) \in H_{1}\left(T^{2} \times[0,1] ; \mathbb{Z}\right) \cong H_{1}\left(T^{2} ; \mathbb{Z}\right) \cong \mathbb{Z}^{2} .
$$


Moreover, each sign is realized by a unique (up to isotopy) basic slice. Applying the diffeomorphism

$$
T^{2} \times[0,1] \longrightarrow T^{2} \times[0,1]
$$

given by

$$
\left(\begin{array}{rr}
1 & 2-p \\
-1 & p-1
\end{array}\right)
$$

we obtain a basic slice with boundary slopes -1 and $-\frac{p}{p-1}$ which is diffeomorphic to $\left(S^{1} \times D^{2} \backslash N, \eta\right)$, together with a section $v$ of $\eta$ which is nowhere zero at the boundary, tangent to it, and with zero locus a smooth curve homologous to $\pm(2-p, p-1)$. The section $v$ can be assumed to coincide with the vector field $\frac{\partial}{\partial z}$ on $\partial N$ in the above coordinates $(z,(x, y))$. Since $\frac{\partial}{\partial z}$ is a nowhere zero section of $\eta$ on $N$, this implies that $v$ extends as a section of $\eta$ on $S^{1} \times D^{2}$ with the stated properties. By choosing the appropriate basic slice one can construct $\eta$ with either choice of sign in Formula (2.2).

Definition 2.5 Let $\eta_{0}$ (respectevely $\eta_{1}$ ) be a tight contact structure on $S^{1} \times$ $D^{2}$ as in the conclusion of Lemma 2.4, satisfying condition (2.2) with the positive (respectively negative) sign. Let $n \geq 2 g$, and define $\xi_{0}$ (respectively $\xi_{1}$ ) to be the contact structure on $Y_{g, n}, n \geq 2 g$, given by (2.1) with $\eta$ replaced by $\eta_{0}$ (respectively $\eta_{1}$ ).

Remark 2.6 By Theorem 2.3 the contact structures $\xi_{0}$ and $\xi_{1}$ of Definition 2.5 are isotopic when $n=2 g$. By the classification from [15], $\xi_{0}$ is not isotopic to $\xi_{1}$ when $n>2 g$. In fact, $\xi_{0}$ and $\xi_{1}$ are not even homotopic (see Remark 3.7 below).

\section{Calculations of $\operatorname{Spin}^{c}$ structures}

The goal of this section is to determine the $\operatorname{Spin}^{c}$ structures $\mathbf{t}_{\xi_{i}}$ induced by the contact structures $\xi_{i}$ of Definition 2.5. We begin with a short review about Spin and $S_{p i n}^{c}$ structures in general. Then, we study Spin and $\operatorname{Spin}^{c}$ structures on disk and circle bundles over surfaces. The section ends with the calculation of $\mathbf{t}_{\xi_{0}}$ and $\mathbf{t}_{\xi_{1}}$.

\section{Generalities on Spin and Spin ${ }^{c}$ structures}

Let $X$ be a smooth, oriented $n$-dimensional manifold, $n \geq 3$. The structure group of its tangent frame bundle $P_{X}$ can be reduced to $S O(n)$ by e.g. introducing a Riemannian metric on $X$. A Spin structure on $X$ is a principal 
$\operatorname{Spin}(n)$-bundle $P_{\operatorname{Spin}(n)} \rightarrow X$ such that $P_{X}$ is isomorphic to the associated bundle

$$
P_{\operatorname{Spin}(n)} \times{ }_{\rho} S O(n),
$$

where

$$
\rho: \operatorname{Spin}(n) \rightarrow S O(n)
$$

is the universal covering map. A Spin structure on $X$ exists if and only if the second Stiefel-Whitney class $w_{2}(X)$ vanishes. In this case, the set of Spin structures is a principal homogeneous space on $H^{1}(X ; \mathbb{Z} / 2 \mathbb{Z})$.

The quotient of $\operatorname{Spin}(n) \times S^{1}$ modulo the subgroup

$$
\{ \pm(1,1)\} \cong \mathbb{Z} / 2 \mathbb{Z}
$$

is, by definition, the group $\operatorname{Spin}^{c}(n)$. There are two canonical surjective homomorphisms

$\rho_{1}: \operatorname{Spin}^{c}(n) \rightarrow \operatorname{Spin}(n) /\{ \pm 1\}=S O(n), \quad \rho_{2}: \operatorname{Spin}^{c}(n) \rightarrow S^{1} /\{ \pm 1\}=S^{1}$.

A $\operatorname{Spin}^{c}$ structure on $X$ is a principal $\operatorname{Spin}^{c}(n)$-bundle $P_{\operatorname{Spin}^{c}(n)}$ such that

$$
P_{X} \cong P_{\operatorname{Spin}^{c}(n)} \times_{\rho_{1}} S O(n) .
$$

Let $\operatorname{Spin}^{c}(X)$ denote the (possibly empty) set of $\operatorname{Spin}^{c}$ structures on $X$. An element

$$
\mathbf{s}=P_{\operatorname{Spin}^{c}(n)} \in \operatorname{Spin}^{c}(X)
$$

naturally induces a principal $S^{1}$-bundle $P_{S p i n^{c}(n)} \times{ }_{\rho_{2}} S^{1}$. Let $c_{1}(\mathbf{s}) \in H^{2}(X ; \mathbb{Z})$ be the first Chern class of the corresponding complex line bundle. A manifold $X$ admits a Spin ${ }^{c}$ structure if and only if $w_{2}(X)$ has an integral lift, and in fact the set

$$
\left\{c_{1}(\mathbf{s}) \mid \mathbf{s} \in \operatorname{Spin}^{c}(X)\right\} \subset H^{2}(X ; \mathbb{Z})
$$

is the preimage of $w_{2}(X)$ under the natural map

$$
H^{2}(X ; \mathbb{Z}) \rightarrow H^{2}(X ; \mathbb{Z} / 2 \mathbb{Z}) .
$$

Moreover, $\operatorname{Spin}^{c}(X)$ is a principal homogeneous space on $H^{2}(X ; \mathbb{Z})$, and $c_{1}(\mathbf{s}+$ $\alpha)=c_{1}(\mathbf{s})+2 \alpha$ for every

$$
(\mathbf{s}, \alpha) \in \operatorname{Spin}^{c}(X) \times H^{2}(X ; \mathbb{Z}) .
$$

The group $\operatorname{Spin}(n)$ naturally embeds into $\operatorname{Spin}^{c}(n)$, so a $\operatorname{Spin}$ structure induces a $\operatorname{Spin}^{c}$ structure. Moreover, since

$$
\operatorname{Spin}(n)=\operatorname{ker} \rho_{2} \subset \operatorname{Spin}^{c}(n),
$$

a $\operatorname{Spin}^{c}$ structure $\mathbf{s}$ is induced by a Spin structure if and only if $c_{1}(\mathbf{s})=0$. 
Since

$$
\rho_{1}^{-1}(\{1\} \times S O(n))=\operatorname{Spin}^{c}(n) \subset \operatorname{Spin}^{c}(n+1),
$$

if $\operatorname{dim} Y=n$ and $Y=\partial X$, there is a restriction map $\operatorname{Spin}^{c}(X) \rightarrow \operatorname{Spin}^{c}(Y)$. Clearly, this map sends Spin structures to Spin structures.

An oriented two-plane field $\xi$ (and so a contact structure) on a closed, oriented three-manifold $Y$ reduces the structure group of $T Y$ to $U(1) \subset S O(3)$. Since the inclusion $U(1) \subset S O(3)$ admits a canonical lift to $U(2)=\operatorname{Spin}^{c}(3)$, there is a $\operatorname{Spin}^{c}$ structure $\mathbf{t}_{\xi} \in \operatorname{Spin}^{c}(Y)$ canonically associated to $\xi$. The $\operatorname{Spin}^{c}$ structure $\mathbf{t}_{\xi}$ depends only on the homotopy class of $\xi$ as an oriented tangent two-plane field.

\section{Disk bundles}

Let $\Sigma_{g}$ be a closed, oriented surface of genus $g \geq 1$. Let $\pi: D_{g, n} \rightarrow \Sigma_{g}$ be an oriented 2-disk bundle over $\Sigma_{g}$ with Euler number $n$.

By e.g. fixing a metric on $D_{g, n}$ one sees that the tangent bundle of $D_{g, n}$ is isomorphic to the direct sum of the pull-back of $T \Sigma_{g}$ and the vertical tangent bundle, which is isomorphic to the pull-back of the real oriented two-plane bundle $E_{g, n} \rightarrow \Sigma_{g}$ with Euler number $n$. In short, we have

$$
T D_{g, n} \cong \pi^{*}\left(T \Sigma_{g} \oplus E_{g, n}\right) .
$$

Therefore, the structure group of $T D_{g, n}$ can be reduced to $U(2) \subset S O(4)$, which admits a natural lift

$$
A \mapsto\left(\left(\begin{array}{cc}
\operatorname{det} A & 0 \\
0 & 1
\end{array}\right), A\right)
$$

to

$$
\operatorname{Spin}^{c}(4)=\{(A, B) \in U(2) \times U(2) \mid \operatorname{det} A=\operatorname{det} B\} .
$$

Denote by $\mathbf{s}_{0}$ the induced $\operatorname{Spin}^{c}$ structure on $D_{g, n}$. The orientation on $D_{g, n}$ determines an isomorphism $H^{2}\left(D_{g, n} ; \mathbb{Z}\right) \cong \mathbb{Z}$, so the set

$$
\operatorname{Spin}^{c}\left(D_{g, n}\right)=\mathbf{s}_{0}+H^{2}\left(D_{g, n} ; \mathbb{Z}\right)
$$

can be canonically identified with the integers. We denote by

$$
\mathbf{s}_{e}=\mathbf{s}_{0}+e \in \operatorname{Spin}^{c}\left(D_{g, n}\right)
$$

the element corresponding to the integer $e \in \mathbb{Z} \cong H^{2}\left(D_{g, n} ; \mathbb{Z}\right)$.

Lemma 3.1 (a) If $n$ is odd, $D_{g, n}$ admits no Spin structure.

(b) If $n$ is even, $D_{g, n}$ carries Spin structures. Every Spin structure on $D_{g, n}$ induces the Spin $^{c}$ structure $\mathbf{s}_{g-\frac{n}{2}-1}$. 
Proof In view of (3.1) we have $c_{1}\left(\mathbf{s}_{0}\right)=2-2 g+n$, hence

$$
c_{1}\left(\mathbf{s}_{e}\right)=c_{1}\left(\mathbf{s}_{0}\right)+2 e=2(1-g+e)+n .
$$

Since each $c_{1}\left(\mathbf{s}_{e}\right)$ reduces modulo 2 to $w_{2}\left(D_{g, n}\right), D_{g, n}$ admits a Spin structure if and only if $n$ is even. Solving the equation $c_{1}\left(\mathbf{s}_{e}\right)=0$ for $e$ yields the statement.

\section{Circle bundles}

Consider $Y_{g, n}=\partial D_{g, n}$. We have

$$
H_{1}\left(Y_{g, n} ; \mathbb{Z}\right) \cong H^{2}\left(Y_{g, n} ; \mathbb{Z}\right) \cong \mathbb{Z}^{2 g} \oplus \mathbb{Z} / n \mathbb{Z},
$$

where the summand $\mathbb{Z} / n \mathbb{Z}$ is generated by the Poincare dual $F$ of the class of a fiber of the projection $\pi: Y_{g, n} \rightarrow \Sigma_{g}$. Each $\operatorname{Spin}^{c}$ structure $\mathbf{s}_{e} \in \operatorname{Spin}^{c}\left(D_{g, n}\right)$ determines by restriction a $\operatorname{Spin}^{c}$ structure $\mathbf{t}_{e} \in \operatorname{Spin}^{c}\left(Y_{g, n}\right)$. We have

$$
\mathbf{t}_{e}=\mathbf{t}_{0}+e F, \quad e \in \mathbb{Z} .
$$

Since $n F=0$, we see that $\mathbf{t}_{e+n}=\mathbf{t}_{e}$ for every $e$. Therefore, $\mathbf{t}_{0}, \ldots, \mathbf{t}_{n-1}$ is a complete list of torsion Spin ${ }^{c}$ structures on $Y_{g, n}$, i.e. Spin ${ }^{c}$ structures on $Y_{g, n}$ with torsion first Chern class. Notice that for $n$ even different $\operatorname{Spin}^{c}$ structures might have coinciding first Chern classes; for $n$ odd, $c_{1}\left(\mathbf{t}_{i}\right)$ determines $\mathbf{t}_{i}$.

Remark 3.2 The pull-back of $E_{g, n}$ is trivial when restricted to the complement of the zero section, therefore we have

$$
\left.T Y_{g, n} \cong \underline{\mathbb{R}} \oplus \pi^{*}\left(T \Sigma_{g}\right) \subset T D_{g, n}\right|_{Y_{g, n}} \cong \underline{\mathbb{C}} \oplus \pi^{*}\left(T \Sigma_{g}\right),
$$

where $\underline{\mathbb{R}}$ and $\underline{\mathbb{C}}$ are, respectively, the trivial real and complex line bundles. This shows that

$$
\mathbf{t}_{0}=\left.\mathbf{s}_{0}\right|_{Y_{g, n}}=\mathbf{t}_{\zeta},
$$

where $\zeta \subset T Y_{g, n}$ is any oriented tangent two-plane field transverse to the fibers of $\pi: Y_{g, n} \rightarrow \Sigma_{g}$.

Lemma 3.3 (a) If $n$ is odd, $\mathbf{t}_{g-1}$ is the only torsion Spin ${ }^{c}$ structure on $Y_{g, n}$ induced by a Spin structure.

(b) If $n$ is even, $\mathbf{t}_{g-1}$ and $\mathbf{t}_{g+\frac{n}{2}-1}$ are the only torsion Spinc structures on $Y_{g, n}$ induced by a Spin structure. 
Proof Since $c_{1}\left(\mathbf{s}_{0}\right)$ restricts to $H^{2}\left(Y_{g, n} ; \mathbb{Z}\right)$ as $(2-2 g) F$, we have

$$
c_{1}\left(\mathbf{t}_{e}\right)=2(1-g+e) F .
$$

Solving the equation $c_{1}\left(\mathbf{t}_{e}\right)=0$ yields the statement.

Remark 3.4 The $\operatorname{Spin}^{c}$ structure $\mathbf{t}_{g-1}$ on $Y_{g, n}$ is (by definition) the restriction of a Spin $^{c}$ structure on $D_{g, n}$. Although $\mathbf{t}_{g-1}$ is induced by a Spin structure on $Y_{g, n}$, by Lemma $3.1 \mathbf{t}_{g-1}$ does not extend as a Spin structure to $D_{g, n}$ when $n>0$. On the other hand, when $n$ is even the $\operatorname{Spin}^{c}$ structure $\mathbf{t}_{g+\frac{n}{2}-1}$ is induced by a Spin structure on $Y_{g, n}$ which is the restriction of a Spin structure on $D_{g, n}$.

\section{Calculations}

Let $Y$ be a closed, oriented three-manifold and let $\xi \subset T Y$ be an oriented tangent two-plane field. The $\operatorname{Spin}^{c}$ structure $\mathbf{t}_{\xi} \in \operatorname{Spin}^{c}(Y)$ determined by $\xi$ can be also defined as follows [13]. Using a trivialization of $T Y$, the oriented two-plane bundle $\xi$ can be realized as the pull-back of the tangent bundle to the two-sphere $S^{2}$ under a smooth map $Y \rightarrow S^{2}$. This implies, in particular, that the Euler class of $\xi$ is always even. Therefore $\xi$ has a section $v$ which vanishes along a link $L_{v} \subset Y$ with multiplicity two. Being a non-zero section of $\left.T Y\right|_{Y \backslash L_{v}}, v$ determines a trivialization and so a Spin structure on $Y \backslash L_{v}$. Since $v$ vanishes with multiplicity two along $L_{v}$, this Spin structure extends uniquely to a Spin structure on $Y$, which induces a $\operatorname{Spin}^{c}$ structure $\mathbf{t}_{v} \in \operatorname{Spin}^{c}(Y)$. The link $L_{v}$ carries a natural orientation such that $2 \mathrm{PD}\left(\left[L_{v}\right]\right)$ equals the Euler class of $\xi$. According to [13] the $\operatorname{Spin}^{c}$ structure $\mathbf{t}_{\xi}$ is given by

$$
\mathbf{t}_{\xi}=\mathbf{t}_{v}+\operatorname{PD}\left(\left[L_{v}\right]\right) \text {. }
$$

Lemma 3.5 Let $n \geq 2 g$, and let $\xi_{0}$ and $\xi_{1}$ be the contact structures on $Y_{g, n}$ given by Definition 2.5. Let $F \in H^{2}\left(Y_{g, n} ; \mathbb{Z}\right)$ denote the Poincare dual of the homology class of a fiber of the fibration $\pi: Y_{g, n} \rightarrow \Sigma_{g}$. Then, the Euler class of $\xi_{i}, i \in\{0,1\}$, as an oriented two-plane bundle is equal to

$$
(-1)^{i+1} 2 g F \text {. }
$$

If $n$ is even, then $\xi_{i}$ admits a section $v$ vanishing with multiplicity two along a smooth curve $L_{v} \subset Y_{g, n}$ with

$$
P D\left(\left[L_{v}\right]\right)=(-1)^{i}\left(\frac{n}{2}-g\right) F .
$$

Moreover, the Spin structure $\mathbf{t}_{v} \in \operatorname{Spin}\left(Y_{g, n}\right)$ is equal to $\mathbf{t}_{g+\frac{n}{2}-1}$. 
Proof The contact structure $\zeta$ on $Y_{g, 2 g-2}$ given by Lemma 2.2 is tangent to the fibers of the fibration $Y_{g, 2 g-2} \rightarrow \Sigma_{g}$. Therefore, any nowhere vanishing vector field tangent to the fibers gives a nowhere zero section $v$ of $\zeta$. By the construction (2.1) defining $\xi_{i}$, this gives a nowhere vanishing section $v$ of $\left.\xi_{i}\right|_{Y_{g, 2 g-2} \backslash U}$, which glues up to the section given in (2) of Lemma 2.4. Therefore, by Definition 2.5 the Euler class of $\xi_{i}$ is

$$
(-1)^{i}(p-1) F=(-1)^{i}(n-2 g) F=(-1)^{i+1} 2 g F .
$$

If $n$ is even, then $p-1=n-2 g$ is even as well. In this case we may assume that the section given in (2) of Lemma 2.4 vanishes with multiplicity two along a smooth curve representing $(-1)^{i}\left(\frac{p-1}{2}\right)=(-1)^{i}\left(\frac{n}{2}-g\right)$ times the homology class of the core circle. Splicing such a section to the nowhere zero section $v$ we obtain the formula for $P D\left(\left[L_{v}\right]\right)$.

We see from Equation (3.2) that if $v$ is the non-vanishing section of $\zeta$ used above, we have $\mathbf{t}_{v}=\mathbf{t}_{\zeta}$. On the other hand, by Remark $3.2 \mathbf{t}_{\zeta}$ is equal to $\mathbf{t}_{0}$, which extends as a Spin structure to $D_{g, n}$ by definition. Thus, the Spin structure $\mathbf{t}_{v} \in \operatorname{Spin}\left(Y_{g, n}\right)$ extends to $D_{g, n}$ away from the preimage $\pi^{-1}\left(D^{2}\right)$ of some two-disk $D^{2} \subset \Sigma_{g}$. But $\pi^{-1}\left(D^{2}\right)$ is homeomorphic to a ball, therefore the unique Spin structure on $\partial \pi^{-1}\left(D^{2}\right)$ extends for trivial reasons to the unique Spin structure on $\pi^{-1}\left(D^{2}\right)$. This proves that $\mathbf{t}_{v}$ extends to $D_{g, n}$ as a Spin structure. Therefore, by Remark $3.4 \mathbf{t}_{v}$ must coincide with $\mathbf{t}_{g+\frac{n}{2}-1}$.

Proposition 3.6 Let $n \geq 2 g$ and let $\xi_{0}$ and $\xi_{1}$ be the contact structures on $Y_{g, n}$ from Definition 2.5. Then, $\mathbf{t}_{\xi_{0}}=\mathbf{t}_{n-1}$ and $\mathbf{t}_{\xi_{1}}=\mathbf{t}_{2 g-1}$.

Proof By Lemma 3.5, the Euler class of $\xi_{i}$ coincides with $c_{1}\left(\mathbf{t}_{2 i g-1}\right), i=0,1$. If $n$ is odd, $H^{2}\left(Y_{g, n} ; \mathbb{Z}\right)$ has no 2 -torsion and the result follows. If $n$ is even, by Lemma 3.5 and Equation (3.2) we have

$$
\mathbf{t}_{\xi_{i}}=\mathbf{t}_{g+\frac{n}{2}-1}+(-1)^{i}\left(\frac{n}{2}-g\right) F=\mathbf{t}_{2 i g-1} .
$$

Remark 3.7 Since the Spin $^{c}$ structures $\mathbf{t}_{\xi_{i}}$ are homotopy invariants, Proposition 3.6 implies that $\xi_{0}$ and $\xi_{1}$ are not homotopic as oriented tangent two-plane fields on $Y_{g, n}$ once $n>2 g$. It can be shown that $\xi_{0}$ and $\xi_{1}$ are contactomorphic, i.e. there is a self-diffeomorphism of $Y_{g, n}$ sending one to the other. We will not use that fact in this paper. 


\section{Monopole equations and the proof of Theorem 1.1}

This section is devoted to the proof of the main result of the paper, Theorem 1.1. For definitions and properties of the solutions to the Seiberg-Witten equations on cylinders $\mathbb{R} \times Y$ and on symplectic fillings, we refer the reader to [20] and [16].

Lemma 4.1 Let $n \geq 2 g$, and let $\xi_{i}, i \in\{0,1\}$ be one of the contact structures on $Y_{g, n}$ from Definition 2.5. Then,

(1) The moduli space $N\left(Y_{g, n}, \mathbf{t}_{\xi_{i}}\right)$ of solutions to the unperturbed SeibergWitten equations is smooth and consists of reducibles.

(2) There exists a real number $\epsilon>0$ such that, if $\mu \in \Omega^{2}\left(Y_{g, n}\right)$ is a closed two-form whose $L^{2}$-norm is smaller than $\epsilon$, then either

$$
[\mu]=2 \pi c_{1}\left(\mathbf{t}_{\xi_{i}}\right) \in H^{2}\left(Y_{g, n} ; \mathbb{R}\right)
$$

or the $\mu$-perturbed Seiberg-Witten moduli space $N_{\mu}\left(Y_{g, n}, \mathbf{t}_{\xi_{i}}\right)$ is empty.

Proof By Theorems 1 and 2 of [20] (see also [21, Theorem 2.2]), if $|n|>$ $2 g-2$ and $e \notin[0,2 g-2]$, then the moduli space $N\left(Y_{g, n}, \mathbf{t}_{e}\right)$ is smooth and contains only reducibles. Therefore, part (1) of the statement follows from Proposition 3.6.

To prove (2) we argue by contradiction. Let $\left\{\mu_{n}\right\}_{n=1}^{\infty} \subset \Omega^{2}\left(Y_{g, n}\right)$ be a sequence of closed two-forms such that $\left[\mu_{n}\right] \neq 2 \pi c_{1}\left(\mathbf{t}_{\xi_{i}}\right),\left\|\mu_{n}\right\| \stackrel{n \rightarrow \infty}{\rightarrow} 0$, and $N_{\mu_{n}}\left(Y, \mathbf{t}_{\xi_{i}}\right)$ contains some element $\left[\left(A_{n}, \psi_{n}\right)\right]$. By a standard compactness argument there is a subsequence converging, modulo gauge, to a solution $\left(A_{0}, \psi_{0}\right)$ of the unperturbed Seiberg-Witten equations, which is reducible by part (1). We have $\psi_{n} \neq 0$ because the assumption $\left[\mu_{n}\right] \neq 2 \pi c_{1}\left(\mathbf{t}_{\xi_{i}}\right)$ implies that $\left(A_{n}, \psi_{n}\right)$ must be irreducible, so we can set $\varphi_{n}=\frac{\psi_{n}}{\left\|\psi_{n}\right\|}$ for every $n$.

The smoothness of $N\left(Y_{g, n}, \mathbf{t}_{\xi_{i}}\right)$ implies that the kernel of the Dirac operator $D_{A_{0}}$ is trivial, so by standard elliptic estimates (see e.g. [4, page 423]) we have

$$
1=\left\|\varphi_{n}\right\| \leq C\left\|D_{A_{0}} \varphi_{n}\right\|
$$

for some constant $C$. On the other hand, since the $\left(A_{n}, \psi_{n}\right)$ 's are solutions to the Seiberg-Witten equations, by writing $A_{n}=A_{0}+a_{n}$ we have

$$
0=D_{A_{n}} \varphi_{n}=D_{A_{0}} \varphi_{n}+a_{n} \cdot \varphi_{n},
$$

where '.' denotes Clifford multiplication. Since $\left\|\varphi_{n}\right\|=1$ while $\left\|a_{n}\right\| \rightarrow 0$, this implies $D_{A_{0}} \varphi_{n} \rightarrow 0$, contradicting (4.1). 
Proposition 4.2 Let $n \geq 2 g$, and let $\xi_{i}, i \in\{0,1\}$ be one of the contact structures on $Y_{g, n}$ from Definition 2.5. Let $(W, \omega)$ be a weak symplectic semifilling of $\left(Y_{g, n}, \xi_{i}\right)$. Then $\partial W$ is connected, $b_{2}^{+}(W)=0$ and the homomorphism

$$
H^{2}(W ; \mathbb{R}) \rightarrow H^{2}(\partial W ; \mathbb{R})
$$

induced by the inclusion $\partial W \subset W$ is the zero map.

Proof In [17] it is proved that if $(W, \omega)$ is a weak semi-filling of a contact three-manifold $(Y, \xi)$, where $Y$ carries metrics with positive scalar curvature, then (a) $\partial W$ is connected and $b_{2}^{+}(W)=0([17$, Theorem 1.4]) and (b) the homomorphism

$$
H^{2}(W ; \mathbb{R}) \rightarrow H^{2}(\partial W ; \mathbb{R})
$$

induced by the inclusion $\partial W \subset W$ is the zero map ([17, Proposition 2.1]).

The positive scalar curvature assumption was used in the proof of $[17$, Theorem 1.4] to guarantee that the moduli space $N\left(Y, \mathbf{t}_{\xi}\right)$ of solutions to the unperturbed Seiberg-Witten equations is smooth and consists of reducibles. But this is true for the contact structures $\xi_{0}$ and $\xi_{1}$ on $Y_{g, n}$ by part (1) of Lemma 4.1. Therefore, conclusion (a) holds for any symplectic semi-filling of $\left(Y_{g, n}, \xi_{i}\right), i \in\{0,1\}$.

Similarly, the existence of positive scalar curvature metrics was used in $[17$, Proposition 2.1] to prove that if $\mu$ is a closed two-form whose $L^{2}$-norm is sufficiently small and $[\mu] \neq 2 \pi c_{1}\left(\mathbf{t}_{\xi}\right)$, then the $\mu$-perturbed Seiberg-Witten moduli space $N_{\mu}\left(Y, \mathbf{t}_{\xi}\right)$ is empty. But for the contact structures $\xi_{0}$ and $\xi_{1}$ this is precisely the content of (2) in Lemma 4.1. Hence, conclusion (b) holds for symplectic semi-fillings of $\left(Y_{g, n}, \xi_{i}\right), i \in\{0,1\}$.

We shall now give a purely topological argument showing that, under some restrictions on $g$ and $n$, there is no smooth four-manifold $W$ with $\partial W=Y_{g, n}$ satifying the conclusion of Proposition 4.2.

Let $\mathbb{C P}^{2}$ be the complex projective plane. Denote by $\widehat{\mathbb{C P}^{2}}$ the blow-up of $\mathbb{C P}^{2}$ at $k$ distinct points. The second homology group $H_{2}\left(\widehat{\mathbb{C P}^{2}} ; \mathbb{Z}\right)$ is generated by classes $h, e_{1}, e_{2}, \ldots, e_{k}$, where $h$ corresponds to the standard generator of $H_{2}\left(\mathbb{C P}^{2} ; \mathbb{Z}\right)$ and the $e_{i}$ 's are the classes of the exceptional curves.

Let $d$ be a positive integer, and suppose $k \geq 2 d$. Define $\boldsymbol{\Lambda}_{d}=\left(H_{d}, Q_{d}\right)$ as the intersection lattice given by the subgroup

$$
H_{d}=\left\langle e_{1}-e_{2}, e_{2}-e_{3}, \ldots, e_{2 d-1}-e_{2 d}, h-e_{1}-e_{2}-\ldots-e_{d}\right\rangle \subset H_{2}\left(\widehat{\mathbb{C P}^{2}} ; \mathbb{Z}\right)
$$


together with the restriction $Q_{d}$ of the intersection form $Q_{\widehat{\mathbb{C P}^{2}}}$. For $m \geq 1$ let $\mathbf{D}_{m}=\left(\mathbb{Z}^{m}, m(-1)\right)$ be that standard negative definite diagonal lattice. The following lemma generalizes a result from [18].

Lemma 4.3 $\boldsymbol{\Lambda}_{d}$ does not embed into $\mathbf{D}_{m}$.

Proof Arguing by contradiction, suppose that $j: \boldsymbol{\Lambda}_{d} \hookrightarrow \mathbf{D}_{m}$ is an embedding. Then,

$$
\operatorname{rk} \mathbf{D}_{m}=m \geq \operatorname{rk} \boldsymbol{\Lambda}_{d}=2 d .
$$

Let $w_{1}, \ldots, w_{2 d}$ be the obvious generators of $\boldsymbol{\Lambda}_{d}$ with $w_{i} \cdot w_{i}=-2$ for $i \leq 2 d-1$ and $w_{2 d} \cdot w_{2 d}=-(d-1)$. Then, since the elements $w_{1}, \ldots, w_{2 d-1}$ have square -2 , there is a set $e_{1}, \ldots, e_{m}$ of standard generators of $\mathbf{D}_{m}$ such that

$$
j\left(w_{i}\right)=e_{i}-e_{i+1}, \quad i=1, \ldots, 2 d-1 .
$$

Suppose that

$$
j\left(w_{2 d}\right)=a_{1} e_{1}+\cdots+a_{m} e_{m}, \quad a_{1}, \ldots, a_{m} \in \mathbb{Z} .
$$

Then, the relations $w_{2 d} \cdot w_{i}=0$ for $i \neq d$ and $w_{2 d} \cdot w_{d}=1$ imply

$$
j\left(w_{2 d}\right)=a \sum_{i=1}^{2 d} e_{i}+\sum_{i=d+1}^{2 d} e_{i}+\sum_{i \geq 2 d+1} a_{i} e_{i},
$$

for some $a \in \mathbb{Z}$, therefore $j\left(w_{2 d}\right) \cdot j\left(w_{2 d}\right) \leq-d$, which is impossible because $w_{2 d} \cdot w_{2 d}=-(d-1)$.

Proposition 4.4 Suppose that $2 g \geq d(d+1)$ and $n \leq(d+1)^{2}+3$ for some positive integer $d$. Then, there is no smooth, compact, oriented four-manifold $W$ such that $\partial W=Y_{g, n}, b_{2}^{+}(W)=0$ and the map

$$
H^{2}(W ; \mathbb{R}) \rightarrow H^{2}(\partial W ; \mathbb{R})
$$

is the zero map.

Proof Consider a smooth curve $C \subset \mathbb{C P}^{2}$ of degree $d+2$, and let $\widehat{\mathbb{C P}^{2}}$ be the blow-up of $\mathbb{C P}^{2}$ at $k$ distinct points of $C$. Let $\widehat{C}$ be the proper trasform of $C$ inside $\widehat{\mathbb{C P}^{2}}$.

Denote by $\widetilde{C} \subset \widehat{\mathbb{C P}^{2}}$ a smooth, oriented surface obtained by adding $g-\frac{1}{2} d(d+1)$ fake handles to $\widehat{C}$. Let $Z \subset \widehat{\mathbb{C P}^{2}}$ be the complement of an open tubular neighborhood of $\widetilde{C}$. The boundary of $Z$ is orientation-reversing diffeomorphic to $Y_{g, n}$, where $n=(d+2)^{2}-k$. Clearly, any $n \leq(d+1)^{2}+3$ can be realized by 
some $k \geq 2 d$, in which case the lattice $\boldsymbol{\Lambda}_{d}$ can be realized as a sublattice of the intersection lattice of $\widehat{\mathbb{C P}^{2}}$. The generators $e_{i}-e_{i+1}$ and $h-e_{1}-\ldots-e_{d}$ of $\boldsymbol{\Lambda}_{d}$ can all be represented by smooth surfaces inside $Z$, so we have an embedding

$$
\Lambda_{d} \subset\left(H_{2}(Z ; \mathbb{Z}), Q_{Z}\right) .
$$

Now we argue by contradiction. Let $W$ be a smooth four-manifold as in the statement. Consider a smooth, closed four-manifold $V$ of the form

$$
W \cup_{Y_{g, n}} Z \text {. }
$$

Since

$$
H^{2}(W ; \mathbb{R}) \rightarrow H^{2}(\partial W ; \mathbb{R})
$$

is zero, by Poincaré duality the map

$$
H_{2}(W, \partial W ; \mathbb{R}) \rightarrow H_{1}(\partial W ; \mathbb{R})
$$

vanishes. This implies that

$$
H_{1}(\partial W ; \mathbb{R}) \rightarrow H_{1}(W ; \mathbb{R})
$$

is injective and by Mayer-Vietoris

$$
H_{2}(V ; \mathbb{R})=H_{2}(W ; \mathbb{R})+H_{2}(Z ; \mathbb{R}) .
$$

Therefore, since $b_{2}^{+}(W)=b_{2}^{+}(Z)=0$, we have $b_{2}^{+}(V)=0$. Donaldson's theorem on the intersection form of closed, definite four-manifolds (see $[2,3])$ implies that the intersection lattice $\left(H_{2}(V ; \mathbb{Z}), Q_{V}\right)$ must be isomorphic to $\mathbf{D}_{b_{2}(V)}$. Thus, the resulting existence of an embedding

$$
\Lambda_{d} \subset\left(H_{2}(Z ; \mathbb{Z}), Q_{Z}\right)
$$

contradicts Lemma 4.3.

Proof of Theorem 1.1 The statement follows from Propositions 4.2 and 4.4.

Remark 4.5 The restrictions on $n$ and $g$ appearing in the statement of Proposition 4.4 are only due to the inability of the authors to construct more smooth four-manifolds with the necessary properties. In fact, using slightly different methods, in [19] we proved that the conclusion of Theorem 1.1 holds for every $n \geq 2 g>0$. 


\section{References}

[1] B Aebisher et al, Symplectic geometry, Progress Math. 124, Birkhäuser, Boston, MA (1994)

[2] S K Donaldson, An application of gauge theory to 4-dimensional topology, Jour. of Diff. Geom. 18 (1983) 279-315

[3] S K Donaldson, The Seiberg-Witten equations and 4-manifold topology, Bull. Amer. Math. Soc. 33 (1996) 45-70

[4] S K Donaldson, P B Kronheimer, Geometry of four-manifolds, Oxford Univ. Press (1990)

[5] Y Eliashberg, Classification of overtwisted contact structures on 3-manifolds, Invent. Math. 98 (1989) 623-637

[6] Y Eliashberg, Filling by holomorphic discs and its applications, London Math. Soc. Lecture Notes Series 151 (1991) 45-67

[7] Y Eliashberg, M Gromov, Convex symplectic manifolds, from: 'Several complex variables and complex geometry', Part 2 (Santa Cruz, CA, 1989), 135-162, Proc. Sympos. Pure Math. 52, Part 2, Amer. Math. Soc. Providence, RI (1991)

[8] J Etnyre, Introductory lectures on contact geometry, Proc. Sympos. Pure Math. 71 (2003) 81-107

[9] J Etnyre, K Honda, Tight contact structures with no symplectic fillings, Invent. Math. 148 (2002) 609-626

[10] E Giroux, Convexité en topologie de contact, Comment. Math. Helv. 66 (1991) 637-677

[11] E Giroux, Structures de contact sur les variétés fibrées en cercles audessus d'une surface, Comment. Math. Helv. 76 (2001) 218-262

[12] E Giroux, Contact structures, linkings and fibrations, talk at the meeting " 4 dimensional Manifolds", Oberwolfach, July 2001

[13] R Gompf, Handlebody constructions of Stein surfaces, Ann. of Math. 148 (1998) 619-693

[14] K Honda, On the classification of tight contact structures, I, Geom. Topol. 4 (2000) 309-368

[15] K Honda, On the classification of tight contact structures, II, J. Differential Geom. 55 (2000) 83-143

[16] P Kronheimer, T Mrowka, Monopoles and contact structures, Invent. Math. 130 (1997) 209-255.

[17] P Lisca, Symplectic fillings and positive scalar curvature, Geom. Topol. 2 (1998) 103-116

[18] P Lisca, On symplectic fillings of 3-manifolds, Proceedings of the $6^{\text {th }}$ Gökova Geometry-Topology Conference, Turkish J. Math. 23 (1999) 151-159 
[19] P Lisca, A Stipsicz, Tight, not semi-fillable contact circle bundles, Math. Ann. to appear, arXiv:math.SG/0211429

[20] T Mrowka, P Ozsváth, B Yu, Seiberg-Witten monopoles on Seifert fibered spaces, Comm. Anal. Geom. 5 (1997) 685-791

[21] P Ozsváth, Z Szabó, On embedding of circle bundles in 4-manifolds, Math. Res. Lett. 7 (2000) 657-669 